Research Article

\title{
Certain Classes of Analytic Functions Bound with Kober Operators in $q$-Calculus
}

\author{
S. D. Purohit $\mathbb{D}^{1},{ }^{1}$ M. M. Gour $\mathbb{D}^{2},{ }^{2}$ S. Joshi $\mathbb{D}^{2},{ }^{2}$ and D. L. Suthar $\left.{ }^{3}\right)^{3}$ \\ ${ }^{1}$ Department of HEAS (Mathematics), Rajasthan Technical University, Kota, India \\ ${ }^{2}$ Department of Mathematics and Statistics, Manipal University Jaipur, Jaipur, India \\ ${ }^{3}$ Department of Mathematics, Wollow University, Dessie Campus, Wollo Dessie, Amhara, Ethiopia
}

Correspondence should be addressed to D. L. Suthar; dlsuthar@gmail.com

Received 7 May 2021; Accepted 11 June 2021; Published 21 June 2021

Academic Editor: V. Ravichandran

Copyright $\odot 2021$ S. D. Purohit et al. This is an open access article distributed under the Creative Commons Attribution License, which permits unrestricted use, distribution, and reproduction in any medium, provided the original work is properly cited.

Through applying the Kober fractional $q$-calculus apprehension, we preliminary implant and introduce new types of univalent analytical functions with a $q$-differintegral operator in the open disk $\mathbb{U}=\{\xi \in \mathbb{C}:|\xi|<1\}$. The coefficient inequality and distortion theorems are among the results examined with these forms of functions. Specific cases are responded and addressed immediately. The findings include an expansion of the numerous established results in the $q$-theory of analytical functions.

\section{Introduction and Preliminary}

The $q$-analysis theory has been applied in recent times in several fields of science and engineering. The fractional $q$-calculus is indeed an analog of the conventional fractional calculus in $q$-theory. Very recently, Wang et al. [1] and Yan et al. [2] investigated the properties of subclasses of multivalent analytic or meromorphic functions expressed with $q$-difference operators. Furthermore, Srivastava [3] investigated the excellent work with $q$-calculus and fractional $q$-calculus operators, which is quite valuable for academics working on these issues. The applications of fractional $q$-calculus operators have been investigated by Purohit and Raina [4] to describe several new classes of analytic functions in open disk $\mathbb{U}=\{\xi \in \mathbb{C}:|\xi|<1\}$. Moreover, Murugusundaramoorthy et al. [5], Purohit [6], and Purohit and Raina [4, 7] gave related work and added various classes of univalent and multivalently analytic functions in open unit disk $\mathbb{U}$. Several others have also released new classes of analytical functions with the resources of $q$-calculus operators. For any more inquiries on the analytic functions classes, we refer to [1, 2, 8-13] for functions described by applying $q$-calculus operators and subject related to this work.
In the current inquiry, we are planning to develop few additional families of analytic functions applying the Kober differential and integral operators in $q$-calculus. The results obtained must also provide the coefficient inequalities and distortion theorems for the subclasses established here below. First, we use the main notations and definitions in the $q$-calculus which are relevant to grasp the object of the study.

For each complex number $\mathfrak{P}$, the $q$-shifted factorials are delimited by

$$
\begin{aligned}
& (\mathfrak{P} ; q)_{m}=\prod_{j=0}^{m-1}\left(1-q^{j} \mathfrak{P}\right) ; \quad m \in \mathbb{N}, \\
& (\mathfrak{P} ; q)_{0}=1,
\end{aligned}
$$

and with regard to the basic analog of the gamma function,

$$
\left(q^{\mathfrak{P}} ; q\right)_{m}=\frac{\Gamma_{q}(\mathfrak{P}+m)(1-q)^{m}}{\Gamma_{q}(\mathfrak{P})} ; \quad m>0,
$$

in which the q-gamma function is set by (see [14])

$$
\Gamma_{q}(\mathfrak{P})=\frac{(q ; q)_{\infty}(1-q)^{1-\mathfrak{P}}}{\left(q^{\mathfrak{P}} ; q\right)_{\infty}} ; \quad 0<q<1
$$


The recurrence relationship specified by Gaspar and Rahman [15] for the q-gamma function is

$$
\Gamma_{q}(1+\mathfrak{P})=\frac{\left(1-q^{\mathfrak{P}}\right) \Gamma_{q}(\mathfrak{P})}{1-q} .
$$

If $|q|<1$, then equation (1) shall continue to play a role $m=\infty$ as an infinite product of convergence:

$$
(\mathfrak{P} ; q)_{\infty}=\prod_{j=0}^{\infty}\left(1-\mathfrak{P} q^{j}\right),
$$

and we have

$$
(\mathfrak{P} ; q)_{m}=\frac{(\mathfrak{P} ; q)_{\infty}}{\left(\mathfrak{P} q^{m} ; q\right)_{\infty}} ; \quad m \in \mathbb{N} \cup\{\infty\} .
$$

The $q$-binomial expansion is now as follows:

$$
(a-b)_{\tau}=a^{\tau}\left(\frac{-b}{a} ; q\right)_{\tau}=a^{\tau}{ }_{1} \phi_{0}\left[q^{-\tau} ;-; q, \frac{b q^{\tau}}{a}\right] .
$$

[15] accounts for Jackson's $q$-integral and $q$-derivative of a function $f$, which are described on a subset of $\mathbb{C}$, as

$$
D_{q} f(\xi)=\frac{f(\xi)-f(q \xi)}{\xi(1-q)} ; \quad \xi \neq 0, q \neq 0,
$$

with

$$
\begin{gathered}
D_{\xi}^{\mu}=\frac{\left(1-q^{\mu}\right) \xi^{\mu-1}}{1-q} \\
\int_{0}^{\xi} f(y) \mathrm{d}_{q} y=\xi(1-q) \sum_{k=0}^{\infty} q^{k} f\left(\xi q^{k}\right) .
\end{gathered}
$$

\section{The Fractional $q$-Calculus Operators}

Purohit and Raina [4] described the fractional $q$-integral operator of function $f(\xi)$ given by

$$
I_{q, \xi}^{\mathfrak{P}} f(\xi)=\frac{1}{\Gamma_{q}(\mathfrak{P})} \int_{0}^{\xi}(\xi-y q)_{\mathfrak{P}-1} f(y) \mathrm{d}_{q} y,
$$

where $\mathfrak{P}>0$ is the order of integral and $f(\xi)$ is an analytic function in $\mathbb{U}$, and $(7)$ the $(\xi-y q)_{\mathfrak{P}-1}$ be expressed as

$$
(\xi-y q)_{\mathfrak{P}-1}=\xi^{\mathfrak{P}-1}{ }_{1} \phi_{0}\left[q^{1-\mathfrak{P}} ;-; q, \frac{y q^{\mathfrak{P}}}{\xi}\right],
$$

where ${ }_{1} \phi_{0}[\mathfrak{P} ;-; q, \xi]$ is special case of basic hypergeometric series ${ }_{2} \phi_{1}[\mathfrak{P} ; \mathfrak{J} ; \gamma ; q, \xi]$ for $\gamma=\mathfrak{J}$ is single valued for $|\arg (\xi)|<\pi$ and $|\xi|<1$ (see [15]).

Purohit and Raina [4] defined the $D_{q, \xi}^{\mathfrak{P}} f(\xi)$ fractional $q$-derivative operator of a function $f(\xi)$ by

$$
D_{q, \xi}^{\mathfrak{P}} f(\xi)=\frac{1}{\Gamma_{q}(1-\mathfrak{P})} D_{q, \xi} \int_{0}^{\xi}(\xi-y q)_{-\mathfrak{P}} f(y) \mathrm{d}_{q} y,
$$

where $0 \leq \mathfrak{P}<1$ and $f(\xi)$ is suitably constrained with $D_{q, \xi}^{-\mathfrak{P}} f(\xi)=I_{q, \xi}^{\mathfrak{P}} f(\xi)$.
The Kober fractional $q$-integral operator for a real valued function $f(x)$ is determined by Garg and Chanchalani [16] as

$$
I_{q}^{\gamma, \mathfrak{P}} f(x)=\frac{x^{-\gamma-\mathfrak{P}}}{\Gamma_{q}(\mathfrak{P})} \int_{0}^{x}(x-y q)_{\mathfrak{P}-1} y^{\gamma} f(y) \mathrm{d}_{q} y,
$$

where $\gamma$ being real or complex and $\mathfrak{P}$ is an absolute order of integration with $\mathfrak{R}(\mathfrak{P})>0$. For $q \longrightarrow 1$, operator $(13)$ is reduced to Kober operator $I^{\gamma, \mathfrak{P}} f(x)$ as defined in [17]. For $\gamma=0$, this operator is converted to Riemann-Liouville fractional $q$-integral operator with a power weight function as $I_{q}^{0, \mathfrak{P}} f(x)=x^{-\mathfrak{P}} I_{q}^{\mathfrak{P}} f(x)$.

The Kober fractional $q$-derivative operator for a real valued function $f(x)$ is detailed by Garg and Chanchalani [16] as

$$
D_{q}^{\gamma, \mathfrak{P}} f(x)=\prod_{j=1}^{m}\left([\gamma+j]_{q}+x q^{\gamma+j} D_{q}\right)\left(I_{q}^{\gamma+\mathfrak{P}, m-\mathfrak{P}} f(x)\right),
$$

where $\mathfrak{P}$ is order of derivative with $\mathfrak{R}(\mathfrak{P})>0$ and $m=[\Re(\mathfrak{P})]+1, m \in \mathbb{N}$. For $q \longrightarrow 1$, operator (14) is reduced to Kober operator $D^{\gamma, \mathfrak{P}} f(x)$ as defined in [17].

We are now defining $q$-calculus operators with a view to applying these operators to the geometric function theory of complex analysis.

Definition 1. Kober fractional $q$-integral operator:

For the function $f(\xi)$, the Kober fractional $q$-integral operator is demarcated by

$$
I_{q}^{\gamma, \mathfrak{P}} f(\xi)=\frac{x^{-\gamma-\mathfrak{P}}}{\Gamma_{q}(\mathfrak{P})} \int_{0}^{\xi}(\xi-y q)_{\mathfrak{P}-1} y^{\gamma} f(y) \mathrm{d}_{q} y,
$$

where $\gamma$ is the real or complex, $\mathfrak{P}$ is an absolute order of integration with $\mathfrak{R}(\mathfrak{P})>0$, and the $q$-binomial $(\xi-y q)_{\mathfrak{P}-1}$ is expressed as in (11).

For $q \longrightarrow 1$, operator $(15)$ is reduced to Kober integral operator $I^{\gamma, \mathfrak{P}} f(\xi)$ as defined in [17].

Definition 2. Kober fractional $q$-derivative operator:

The Kober fractional $q$-derivative operator for the function $f(\xi)$ is demarcated by

$$
D_{q}^{\gamma, \mathfrak{P}} f(\xi)=\prod_{j=1}^{m}\left([\gamma+j]_{q}+\xi q^{\gamma+j} D_{q}\right)\left(I_{q}^{\gamma+\mathfrak{P}, m-\mathfrak{P}} f(\xi)\right),
$$

where $\mathfrak{P}$ is the order of derivative with $\mathfrak{R}(\mathfrak{P})>0$ and $m=[\mathfrak{R}(\mathfrak{P})]+1, m \in \mathbb{N}$. For $q \longrightarrow 1$, operator (16) is reduced to Kober derivative operator $D^{\gamma, \mathfrak{P}} f(\xi)$ as defined in [17].

Under Kober $q$-integral and $q$-derivative operators fixed by (15) and (16), we offer the following image formulae for function $\xi^{\mu}$.

Remark 1. If $\mathfrak{P}, \gamma, \mu \in \mathbb{C}, \quad \mathfrak{R}(\gamma+\mathfrak{P}+\mu+1)>0$, and $\mathfrak{R}(\gamma+\mu+1)>0$, then 


$$
D_{q}^{\gamma, \mathfrak{P}} \xi^{\mu}=\frac{\Gamma_{q}(\gamma+\mathfrak{P}+\mu+1)}{\Gamma_{q}(\gamma+\mu+1)} \xi^{\mu}
$$

Remark 2. If $\mathfrak{P}, \gamma, \mu \in \mathbb{C}, \quad \mathfrak{R}(\gamma+\mu+1)>0, \quad$ and $\mathfrak{R}(\gamma+\mathfrak{P}+\mu+1)>0$, then

$$
I_{q}^{\gamma, \mathfrak{P}} \xi^{\mu}=\frac{\Gamma_{q}(\gamma+\mu+1)}{\Gamma_{q}(\gamma+\mathfrak{P}+\mu+1)} \xi^{\mu} .
$$

\section{New Classes of Functions}

Let $\mathscr{A}_{m}$ represent the function class of the form

$$
f(\xi)=\xi+\sum_{k=m+1}^{\infty} a_{k} \xi^{k} ; \quad m \in \mathbb{N}
$$

which are analytic and univalent in open unit disk $\mathbb{U}$. Above, let $\mathscr{A}_{m}^{-}$highlight the subclass of $\mathscr{A}_{m}$ imposing of analytical and univalent functions articulated in the form

$$
f(\xi)=\xi-\sum_{k=m+1}^{\infty} a_{k} \xi^{k} ; \quad a_{k} \geq 0, m \in \mathbb{N} .
$$

For the dedication of this work, we describe a fractional $q$-differintegral operator $\Omega_{q}^{\gamma, \mathfrak{P}}$ for a function $f(\xi)$ of the form (20) by

$$
\begin{aligned}
\Omega_{q}^{\gamma, \mathfrak{P}} f(\xi) & =\frac{\Gamma_{q}(\gamma+2)}{\Gamma_{q}(\gamma+\mathfrak{P}+2)} \xi^{-1} D_{q}^{\gamma, \mathfrak{P}} f(\xi) \\
& =1-\sum_{k=m+1}^{\infty} \frac{\Gamma_{q}(\gamma+2) \Gamma_{q}(\gamma+\mathfrak{P}+k+1)}{\Gamma_{q}(\gamma+\mathfrak{P}+2) \Gamma_{q}(\gamma+k+1)}, \\
\Omega_{q}^{\gamma, \mathfrak{P}} f(\xi) & =1-\sum_{k=m+1}^{\infty} A(\mathfrak{P}, \gamma, k, q) a_{k} \xi^{k-1},
\end{aligned}
$$

where

$$
A(\mathfrak{P}, \gamma, k, q)=\frac{\Gamma_{q}(\gamma+2) \Gamma_{q}(\gamma+\mathfrak{P}+k+1)}{\Gamma_{q}(\gamma+\mathfrak{P}+2) \Gamma_{q}(\gamma+k+1)},
$$

and $\mathfrak{R}(\gamma+2)>0,0<q<1, \xi \in \mathbb{U}, m \in \mathbb{N}, \mathfrak{R}(\mathfrak{P})>0$, and $D_{q}^{\gamma, \mathfrak{P}} f(\xi)$ represent a fractional $q$-derivative of $f(\xi)$ of order $\mathfrak{P}$. We announce here the alike classes of functions connecting operator (21):

$$
\mathcal{S}_{q}^{\gamma, \mathfrak{P}}(\mathfrak{I})=\left\{f \in \mathscr{A}_{m}^{-},\left|\frac{\Omega_{q}^{\gamma, \mathfrak{P}} f(\xi)-1}{\Omega_{q}^{\gamma, \mathfrak{P}} f(\xi)-2 \mathfrak{T}+1}\right|<\mathfrak{J}\right\}
$$

where

$\mathfrak{R}(\mathfrak{P})>0, \mathfrak{R}(\gamma+2)>0,0 \leq \mathfrak{I}<1,0 \leq \mathfrak{J}<1,0<q<1, \xi \in \mathbb{U}$. And

$$
\underset{q}{\gamma, \mathfrak{P}}(\tau)=\left\{f \in \mathscr{A}_{m}^{-}, \mathfrak{R}\left((1-\tau) \Omega_{q}^{\gamma, \mathfrak{P}} f(\xi)+\tau\left(\frac{1-q^{1-\mathfrak{P}}}{1-q}\right) \Omega_{q}^{\gamma, \mathfrak{P}+1} f(\xi)\right)>\mathfrak{J}\right\}
$$

The subsequent coefficient bounds for functions of the form (20) that belong to the classes $\mathcal{S}_{q}^{\gamma, \mathfrak{P}}(\mathfrak{T})$ and $\underset{q}{\gamma, \mathfrak{P}}(\tau)$ are now obtained (interpreted above).

Theorem 1. A function $f$ defined by (20) is connected to the class $\mathcal{S}_{q}^{\gamma, \mathfrak{P}}(\mathfrak{I})$ if and only if

$$
\sum_{k=m+1}^{\infty} A(\mathfrak{P}, \gamma, k, q) a_{k}(1+\mathfrak{J}) \leq 2 \mathfrak{J}(1-\mathfrak{I})
$$

where

$$
A(\mathfrak{P}, \gamma, k, q)=\frac{\Gamma_{q}(\gamma+2) \Gamma_{q}(\gamma+\mathfrak{P}+k+1)}{\Gamma_{q}(\gamma+\mathfrak{P}+2) \Gamma_{q}(\gamma+k+1)} .
$$

The result is sharp.

Proof. Let us consider that inequality (25) holds, and for $|\xi|=1$, we have

$$
\begin{aligned}
\left|\Omega_{q}^{\gamma, \mathfrak{P}} f(\xi)-1\right|-\mathfrak{J}\left|\Omega_{q}^{\gamma, \mathfrak{P}} f(\xi)-2 \mathfrak{T}+1\right| & =\left|-\sum_{k=m+1}^{\infty} A(\mathfrak{P}, \gamma, k, q) a_{k} \xi^{k-1}\right|-\mathfrak{J}\left|2(1-\mathfrak{T})-\sum_{k=m+1}^{\infty} A(\mathfrak{P}, \gamma, k, q) a_{k} \xi^{k-1}\right| \\
& \leq \sum_{k=m+1}^{\infty} A(\mathfrak{P}, \gamma, k, q) a_{k}(1+\mathfrak{J})-2 \mathfrak{J}(1-\mathfrak{T}) \leq 0,
\end{aligned}
$$


and by our assumption, this indicates that $f(\xi) \in \underset{q}{\mathcal{S}, \mathfrak{P}}(\mathfrak{T})$.

which implies that

For the proof of converse part, suppose that $f(\xi) \in \mathcal{S}_{q}^{\gamma, \mathfrak{P}}(\mathfrak{I})$, and then it follows that

$$
\left|\frac{\Omega_{q}^{\gamma, \mathfrak{P}} f(\xi)-1}{\Omega_{q}^{\gamma, \mathfrak{P}} f(\xi)-2 \mathfrak{T}+1}\right|<\mathfrak{J}
$$

$$
\begin{aligned}
& \left|\frac{\Omega_{q}^{\gamma, \mathfrak{P}} f(\xi)-1}{\Omega_{q}^{\gamma, \mathfrak{P}} f(\xi)-2 \mathfrak{T}+1}\right| \\
& =\left|-\sum_{k=m+1}^{\infty} A(\mathfrak{P}, \gamma, k, q) a_{k} \xi^{k-1}\right|\left|2(1-\mathfrak{T})-\sum_{k=m+1}^{\infty} A(\mathfrak{P}, \gamma, k, q) a_{k} \xi^{k-1}\right|^{-1}<\mathfrak{J} .
\end{aligned}
$$

Since $|\Re(\xi)| \leq|\xi|$ for any $\xi$, therefore on choosing values of $\xi$ on the real axis so that $\Omega_{q}^{\gamma, \mathfrak{P}} f(\xi)$ is real and allowing $\xi \longrightarrow 1$ all through real values, we obtain from above inequality

$$
\sum_{k=m+1}^{\infty} A(\mathfrak{P}, \gamma, k, q) a_{k} \leq 2 \mathfrak{I}(1-\mathfrak{T})-\mathfrak{J} \sum_{k=m+1}^{\infty} A(\mathfrak{P}, \gamma, k, q) a_{k},
$$

which implies that

$$
\sum_{k=m+1}^{\infty} A(\mathfrak{P}, \gamma, k, q) a_{k}(1+\mathfrak{J}) \leq 2 \mathfrak{J}(1-\mathfrak{I})
$$

which is desired result. Here, we notice that assumption (25) of Theorem 1 is sharp and the external function is assumed by

$$
f(\xi)=\xi-\frac{2 \mathfrak{J}(1-\mathfrak{T})}{(1+\mathfrak{J}) A(\mathfrak{P}, \gamma, m+1, q)} \xi^{m+1} ; \quad m \in \mathbb{N},
$$

where $A(\mathfrak{P}, \gamma, k, q)$ is defined in (26).
Theorem 2. A function $f$ defined by (20) is connected to the class $\underset{q}{\mathcal{T}, \mathfrak{P}}(\tau)$ if and only if

$$
\sum_{k=m+1}^{\infty} A(\mathfrak{P}, \gamma, k, q) \mathscr{A}_{k, q}(\mathfrak{P}, \gamma, \tau) a_{k} \leq(1-\mathfrak{J}-\tau)+\tau\left(1-q^{1-\mathfrak{P}}\right),
$$

where

$$
\mathscr{A}_{k, q}(\mathfrak{P}, \gamma, \tau)=\left[(1-\tau)(1-q)+\tau\left(1-q^{1-\mathfrak{P}}\right)\left(\frac{1-q^{\gamma+\mathfrak{P}+k+1}}{1-q^{\gamma+\mathfrak{P}+2}}\right)\right] .
$$

The accomplishment is sharp.

Proof. To prove above theorem, we address the elementary assertion that

$$
\mathfrak{R}(g(\xi)) \geq \mathfrak{J} \Leftrightarrow\{1-\mathfrak{J}+g(\xi)\} \geq\{1+\mathfrak{J}-g(\xi)\} .
$$

Now,

$$
\begin{aligned}
g(\xi) & =(1-\tau) \Omega_{q}^{\gamma, \mathfrak{P}} f(\xi)+\tau\left(\frac{1-q^{1-\mathfrak{P}}}{1-q}\right) \Omega_{q}^{\gamma, \mathfrak{P}+1} f(\xi) \\
& =(1-\tau)\left[1-\sum_{k=m+1}^{\infty} A(\mathfrak{P}, \gamma, k, q) a_{k} \xi^{k-1}\right]+\tau\left(\frac{1-q^{1-\mathfrak{P}}}{1-q}\right)\left[1-\sum_{k=m+1}^{\infty} A(\mathfrak{P}+1, \gamma, k, q) a_{k} \xi^{k-1}\right] \\
& =(1-\tau)+\tau\left(\frac{1-q^{1-\mathfrak{P}}}{1-q}\right)-\frac{1}{(1-q)} \sum_{k=m+1}^{\infty} A_{k, q}(\mathfrak{P}, \gamma, \tau) a_{k} \xi^{k-1}
\end{aligned}
$$

where 


$$
\mathscr{A}_{k, q}(\mathfrak{P}, \gamma, \tau)=\left[(1-\tau)(1-q)+\tau\left(1-q^{1-\mathfrak{P}}\right)\left(\frac{1-q^{\gamma+\mathfrak{P}+k+1}}{1-q^{\gamma+\mathfrak{P}+2}}\right)\right] . \quad \text { In (35), it then suffices to show that }
$$

$$
\begin{gathered}
\left|2-\mathfrak{J}-\tau+\tau\left(\frac{1-q^{1-\mathfrak{P}}}{1-q}\right)-\frac{1}{(1-q)} \sum_{k=m+1}^{\infty} A(\mathfrak{P}, \gamma, k, q) \mathscr{A}_{k, q}(\mathfrak{P}, \gamma, \tau) a_{k} \xi^{k-1}\right| \\
-\left|\mathfrak{J}+\tau-\tau\left(\frac{1-q^{1-\mathfrak{P}}}{1-q}\right)+\frac{1}{(1-q)} \sum_{k=m+1}^{\infty} A(\mathfrak{P}, \gamma, k, q) \mathscr{A}_{k, q}(\mathfrak{P}, \gamma, \tau) a_{k} \xi^{k-1}\right| \geq 0 .
\end{gathered}
$$

Now,

$$
\begin{aligned}
& \left|2-\mathfrak{J}-\tau+\tau\left(\frac{1-q^{1-\mathfrak{P}}}{1-q}\right)-\frac{1}{(1-q)} \sum_{k=m+1}^{\infty} A(\mathfrak{P}, \gamma, k, q) \mathscr{A}_{k, q}(\mathfrak{P}, \gamma, \tau) a_{k} \xi^{k-1}\right| \\
& -\left|\mathfrak{J}+\tau-\tau\left(\frac{1-q^{1-\mathfrak{P}}}{1-q}\right)+\frac{1}{(1-q)} \sum_{k=m+1}^{\infty} A(\mathfrak{P}, \gamma, k, q) \mathscr{A}_{k, q}(\mathfrak{P}, \gamma, \tau) a_{k} \xi^{k-1}\right| \\
& \geq \frac{2}{(1-q)}\left[(1-\mathfrak{J}-\tau)(1-q)+\tau\left(1-q^{1-\mathfrak{P}}\right)-\sum_{k=m+1}^{\infty} A(\mathfrak{P}, \gamma, k, q) \mathscr{A}_{k, q}(\mathfrak{P}, \gamma, \tau) a_{k}|\xi|^{k-1}\right] \geq 0 .
\end{aligned}
$$

This accomplishes the proof of theorem.

We accommodate that answer (33) is sharp. The external function is assumed by

$f(\xi)=\xi-\frac{\left\{(1-\mathfrak{J}-\tau)+\tau\left(1-q^{1-\mathfrak{P}}\right)\right\}}{A(\mathfrak{P}, \gamma, m+1, q) \mathscr{A}_{m+1, q}(\mathfrak{P}, \gamma, \tau)} \xi^{m+1} ; \quad m \in \mathbb{N}$, where $A_{k, q}(\mathfrak{P}, \gamma, \tau)$ is given by (34).

\section{Distortion Theorems}

Theorem 3. Suppose that the function $f$ is defined by (20) in the class $\mathcal{S}_{q}^{\gamma, \mathfrak{P}}(\mathfrak{T})$, then

$$
|\xi|-|\xi|^{m+1}\left(\frac{2 \mathfrak{J}(1-\mathfrak{I})}{1+\mathfrak{J}}\right) B(\mathfrak{P}, \gamma, m, q) \leq|f(\xi)| \leq|\xi|+|\xi|^{m+1}\left(\frac{2 \mathfrak{J}(1-\mathfrak{I})}{1+\mathfrak{J}}\right) B(\mathfrak{P}, \gamma, m, q)
$$

where

$$
B(\mathfrak{P}, \gamma, m, q)=\frac{1}{A(\mathfrak{P}, \gamma, m+1, q)}=\frac{\Gamma_{q}(\gamma+\mathfrak{P}+2) \Gamma_{q}(\gamma+m+2)}{\Gamma_{q}(\gamma+2) \Gamma_{q}(\gamma+\mathfrak{P}+m+2)} .
$$

Furthermore,

$$
|\xi|-|\xi|^{m+1}\left(\frac{2 \mathfrak{J}(1-\mathfrak{T})}{1+\mathfrak{J}}\right) \leq\left|\xi \Omega_{q}^{\gamma, \mathfrak{P}} f(\xi)\right| \leq|\xi|+|\xi|^{m+1}\left(\frac{2 \mathfrak{I}(1-\mathfrak{T})}{1+\mathfrak{J}}\right)
$$


where $\gamma>-2, \mathfrak{R}(\mathfrak{P})>0,0<q<1$.

Proof. Since $f(\xi) \in \mathcal{S}_{q}^{\gamma, \mathfrak{P}}(\mathfrak{I})$, then in interpretation of Theorem 1, we first show that the function

$$
A(\mathfrak{P}, \gamma, k, q)=\frac{\Gamma_{q}(\gamma+2) \Gamma_{q}(\gamma+\mathfrak{P}+k+1)}{\Gamma_{q}(\gamma+\mathfrak{P}+2) \Gamma_{q}(\gamma+k+1)}=\phi(k), \text { (let) }
$$

is an increasing function of $k$ for $\gamma>-2$ and $\mathfrak{R}(\mathfrak{P})>0$.

It follows that

$$
\frac{\phi(k+1)}{\phi(k)}=\frac{\Gamma_{q}(\gamma+\mathfrak{P}+k+2) \Gamma_{q}(\gamma+k+1)}{\Gamma_{q}(\gamma+\mathfrak{P}+k+1) \Gamma_{q}(\gamma+k+2)}=\frac{1-q^{\gamma+\mathfrak{P}+k+1}}{1-q^{\gamma+k+1}}, \quad 0<q<1 .
$$

Taking $k=m+1$, then

$$
\frac{\phi(m+2)}{\phi(m+1)}=\frac{1-q^{\gamma+\mathfrak{p}+m+2}}{1-q^{\gamma+m+2}}, \quad 0<q<1 .
$$

The function $\phi(k)$ is an increasing function of $k$ if $(\phi(m+2) / \phi(m+1)) \geq 1$, and this gives

$$
\frac{1-q^{\gamma+\mathfrak{p}+m+2}}{1-q^{\gamma+m+2}} \geq 1 ; \quad 0<q<1
$$

which implies

$$
q^{\mathfrak{P}} \leq 1, \quad 0<q<1 .
$$

This inequality abides for $\mathfrak{R}(\mathfrak{P})>0$.

Thus, $\phi(k),(k \geq m+1, m \in \mathbb{N})$ is an increasing function of $k$ for $\mathfrak{R}(\gamma+2)>0, \mathfrak{R}(\mathfrak{P})>0$ and $0<q<1$.

Now, (25) gives the alike inequality:

$$
\begin{aligned}
& A(\mathfrak{P}, \gamma, m+1, q) \sum_{k=m+1}^{\infty} a_{k}(1+\mathfrak{J}) \leq \sum_{k=m+1}^{\infty} A(\mathfrak{P}, \gamma, k, q) a_{k}(1 \\
& \quad+\mathfrak{J}) \leq 2 \mathfrak{I}(1-\mathfrak{T}),
\end{aligned}
$$

which implies that

$$
\sum_{k=m+1}^{\infty} a_{k} \leq \frac{2 \mathfrak{J}(1-\mathfrak{I})}{1+\mathfrak{I}} B(\mathfrak{P}, \gamma, m, q),
$$

where $B(\mathfrak{P}, \gamma, m, q)$ is defined in (42), and this last inequality is in the conjunction with the alike inequality (easily obtained from (20)):

$$
|\xi|-|\xi|^{m+1} \sum_{k=m+1}^{\infty} a_{k} \leq|f(\xi)| \leq|\xi|+|\xi|^{m+1} \sum_{k=m+1}^{\infty} a_{k}
$$

and using (50), we have

$$
|\xi|-|\xi|^{m+1}\left(\frac{2 \mathfrak{J}(1-\mathfrak{I})}{1+\mathfrak{J}}\right) B(\mathfrak{P}, \gamma, m, q) \leq|f(\xi)| \leq|\xi|+|\xi|^{m+1}\left(\frac{2 \mathfrak{J}(1-\mathfrak{I})}{1+\mathfrak{I}}\right) B(\mathfrak{P}, \gamma, m, q)
$$

which is result (41) of Theorem 3.
Now, on using (21), we observe that for functions of form (20),

$$
\left|\xi \Omega_{q}^{\gamma, \mathfrak{P}} f(\xi)\right| \geq|\xi|-\sum_{k=m+1}^{\infty} A(\mathfrak{P}, \gamma, k, q) a_{k}|\xi|^{k} \geq|\xi|-|\xi|^{m+1} \sum_{k=m+1}^{\infty} A(\mathfrak{P}, \gamma, k, q) a_{k}
$$

which on using Theorem 1 gives

$$
\left|\xi \Omega_{q}^{\gamma, \mathfrak{P}} f(\xi)\right| \geq|\xi|-2 \mathfrak{J}\left(\frac{1-\mathfrak{T}}{1+\mathfrak{J}}\right)|\xi|^{m+1}
$$

and similarly,

$$
\left|\xi \Omega_{q}^{\gamma, \mathfrak{P}} f(\xi)\right| \leq|\xi|+2 \mathfrak{\Im}\left(\frac{1-\mathfrak{T}}{1+\mathfrak{J}}\right)|\xi|^{m+1},
$$


which implies that

$$
|\xi|-2 \mathfrak{J}\left(\frac{1-\mathfrak{T}}{1+\mathfrak{J}}\right)|\xi|^{m+1} \leq\left|\xi \Omega_{q}^{\gamma, \mathfrak{P}} f(\xi)\right| \leq|\xi|+2 \mathfrak{P}\left(\frac{1-\mathfrak{T}}{1+\mathfrak{J}}\right)|\xi|^{m+1}
$$

Corollary 1. Let the function detailed in (20) be in the class $\mathcal{S}_{q}^{\gamma, \mathfrak{P}}(\mathfrak{I})$, then

$$
\begin{aligned}
\frac{\Gamma_{q}(\gamma+\mathfrak{P}+2)}{\Gamma_{q}(\gamma+2)}\left\{|\xi|-2 \mathfrak{P}\left(\frac{1-\mathfrak{T}}{1+\mathfrak{J}}\right)|\xi|^{m+1}\right\} & \leq\left|D_{q}^{\gamma, \mathfrak{P}} f(\xi)\right| \\
& \leq \frac{\Gamma_{q}(\gamma+\mathfrak{P}+2)}{\Gamma_{q}(\gamma+2)}\left\{|\xi|+2 \mathfrak{J}\left(\frac{1-\mathfrak{T}}{1+\mathfrak{J}}\right)|\xi|^{m+1}\right\},
\end{aligned}
$$

where $\mathfrak{R}(\gamma+2)>0, \mathfrak{R}(\mathfrak{P})>0$, and $\xi \in \mathbb{U}$.

Corollary 2. Let the function detailed in (20) be in the class $\mathcal{S}_{q}^{\gamma, \mathfrak{P}}(\mathfrak{T})$, then

$$
\begin{aligned}
& \frac{\Gamma_{q}(\gamma+2)}{\Gamma_{q}(\gamma+\mathfrak{P}+2)}\left\{|\xi|-2 \mathfrak{J}\left(\frac{1-\mathfrak{T}}{1+\mathfrak{I}}\right)\left(\frac{\Gamma_{q}(\gamma+\mathfrak{P}+2)}{\Gamma_{q}(\gamma+\mathfrak{P}+m+2)}\right)^{2}|\xi|^{m+1}\right\} \leq\left|I_{q}^{\gamma, \mathfrak{P}} f(\xi)\right| \\
& \leq \frac{\Gamma_{q}(\gamma+2)}{\Gamma_{q}(\gamma+\mathfrak{P}+2)}\left\{|\xi|+2 \mathfrak{J}\left(\frac{1-\mathfrak{T}}{1+\mathfrak{I}}\right)\left(\frac{\Gamma_{q}(\gamma+\mathfrak{P}+2)}{\Gamma_{q}(\gamma+\mathfrak{P}+m+2)}\right)^{2}|\xi|^{m+1}\right\},
\end{aligned}
$$

where $\mathfrak{R}(\gamma+2)>0, \mathfrak{R}(\mathfrak{P})>0$, and $\xi \in \mathbb{U}$.

Theorem 4. Suppose that the function $f(\xi)$ detailed in (20) be in the class $\mathscr{T}_{q}^{\gamma, \mathfrak{B}}(\tau)$, then for $\mathfrak{R}(\mathfrak{P})>0, \mathfrak{R}(\gamma+2)>0, \xi \in \mathbb{U}, 0<q<1$,

$|\xi|-B(\gamma, \mathfrak{P}, m, q) C|\xi|^{m+1} \leq|f(\xi)| \leq|\xi|+B(\gamma, \mathfrak{P}, m, q) C|\xi|^{m+1}$,

also

$$
|\xi|-C|\xi|^{m+1} \leq\left|\xi \Omega_{q}^{\gamma, \mathfrak{P}} f(\xi)\right| \leq|\xi|+C|\xi|^{m+1},
$$

where

$$
C=\frac{(1-\mathfrak{J}-\tau)(1-q)+\tau\left(1-q^{1-\mathfrak{P}}\right)}{\mathscr{A}_{m+1, q}(\mathfrak{P}, \gamma, \tau)},
$$

and $\mathscr{A}_{k, q}(\mathfrak{P}, \gamma, \tau)$ and $B(\gamma, \mathfrak{P}, m, q)$ are detailed in (34) and (42), respectively.

Proof. Since $\mathscr{T}_{q}^{\gamma, \mathfrak{P}}(\tau)$, then under the hypothesis of Theorem 2, we have

$$
\sum_{k=m+1}^{\infty} A(\mathfrak{P}, \gamma, k, q) a_{k} \leq \frac{(1-\mathfrak{J}-\tau)(1-q)+\tau\left(1-q^{1-\mathfrak{P}}\right)}{\mathscr{A}_{m+1, q}(\mathfrak{P}, \gamma, \tau)},
$$

which implies that

$$
\sum_{k=m+1}^{\infty} A(\mathfrak{P}, \gamma, k, q) a_{k} \leq C,
$$

where $\mathscr{A}_{k, q}(\mathfrak{P}, \gamma, \tau)$ and $\mathrm{C}$ are given by (34) and (61), respectively, and this last inequality, when combined with the following inequality (which is conveniently obtained from (20)),

$$
|\xi|-|\xi|^{m+1} \sum_{k=m+1}^{\infty} a_{k} \leq|f(\xi)| \leq|\xi|+|\xi|^{m+1} \sum_{k=m+1}^{\infty} a_{k},
$$

and using (63), we have

$$
|\xi|-B(\gamma, \mathfrak{P}, m, q) C|\xi|^{m+1} \leq|f(\xi)| \leq|\xi|+B(\gamma, \mathfrak{P}, m, q) C|\xi|^{m+1},
$$

which is result (59) of Theorem 4.

Now, from (21), we obtain

$$
|\xi|-|\xi|^{m+1} \sum_{k=m+1}^{\infty} A(\lambda, \gamma, k, q) a_{k} \leq\left|\xi \Omega_{q}^{\gamma, \lambda} f(\xi)\right|,
$$

on using (63), this implies that

$$
|\xi|-C|\xi|^{m+1} \leq\left|\xi \Omega_{q}^{\gamma, \lambda} f(\xi)\right|
$$


similarly, we have

$$
\left|\xi \Omega_{q}^{\gamma, \lambda} f(\xi)\right| \leq|\xi|+C|\xi|^{m+1},
$$

and on combining above two results, we have

$$
|\xi|-C|\xi|^{m+1} \leq\left|\xi \Omega_{q}^{\gamma, \lambda} f(\xi)\right| \leq|\xi|+C|\xi|^{m+1}
$$

Corollary 3. Let the function detailed in (20) be in the class $\underset{q}{\gamma}{ }_{q}^{\gamma} \mathfrak{P}(\tau)$, then for all $\xi \in \mathbb{U}, \mathfrak{R}(\gamma+\lambda+2)>0, \mathfrak{R}(\gamma+2)>0$,

$$
\frac{\Gamma_{q}(\gamma+\lambda+2)}{\Gamma_{q}(\gamma+2)}\left\{|\xi|-C|\xi|^{m+1}\right\} \leq\left|D_{q}^{\gamma, \lambda} f(\xi)\right| \leq \frac{\Gamma_{q}(\gamma+\lambda+2)}{\Gamma_{q}(\gamma+2)}\left\{|\xi|+C|\xi|^{m+1}\right\}
$$

The fractional $q$-calculus operators presented in Section 2 may be used to explore numerous different multivalent (or meromorphic) analytic function subclass and geometric characteristics which includes coefficient estimates, distortion bounds, radii of starlikeness, convexity, and so forth. The concept of fractional $q$-calculus can also be used to again with considerations.

\section{Data Availability}

No data were used to support this study.

\section{Conflicts of Interest}

There are no conflicts of interest regarding the publication of this article.

\section{References}

[1] B. Wang, R. Srivastava, R. Srivastava, and J.-L. Liu, "Certain properties of multivalent analytic functions defined by $q$ difference operator involving the Janowski function," AIMS Mathematics, vol. 6, no. 8, pp. 8497-8508, 2021.

[2] C. Yan, R. Srivastava, and J. Liu, "Properties of certain subclass of meromorphic multivalent functions associated with $q$ difference operator," Symmetry, vol. 13, no. 6, p. 1035, 2021.

[3] H. M. Srivastava, "Operators of basic (or $q$-) calculus and fractional $q$-calculus and their applications in geometric function theory of complex analysis," Iranian Journal of Science and Technology. Transaction A: Science, vol. 44, pp. 327-344, 2020.

[4] S. D. Purohit and R. K. Raina, "Certain subclasses of analytic functions associated with fractional q-calculus operators," Mathematica Scandinavica, vol. 109, no. 1, pp. 55-70, 2011.

[5] G. Murugusundaramoorthy, C. Selvaraj, and O. S. Babu, "Subclasses of starlike functions associated with fractional $q$ calculus operators," Journal of Complex Analysis, vol. 2013, Article ID 572718, 8 pages, 2013.

[6] S. D. Purohit, "A new class of multivalently analytic functions associated with fractional $q$-calculus operators," Fractional Differential Calculus, vol. 2, no. 2, pp. 129-138, 2012.

[7] S. D. Purohit and R. K. Raina, "Fractional $q$-calculus and certain subclass of univalent analytic functions," Mathematica, vol. 55, no. 78, pp. 62-74, 2013.

[8] S. Abelman, K. A. Selvakumaran, M. M. Rashidi, and S. D. Purohit, "Subordination conditions for a class of nonbazilevič type defined by using fractional $q$-calculus operators," Facta Universitatis, Series: Mathematics and Informatics, vol. 32, no. 2, pp. 255-267, 2017.
[9] H. Aldweby and M. Darus, "A subclass of harmonic univalent functions associated with $q$-analogue of Dziok-Srivastava operator," ISRN Mathematical Analysis, vol. 2013, Article ID 382312, 6 pages, 2013.

[10] R. K. Saxena, R. K. Yadav, S. D. Purohit, and S. L. Kalla, "Kober fractional $q$-integral operator of the basic analogue of the $H$-function," Revista Técnica de la Facultad de Ingenieria, vol. 28, no. 2, pp. 154-158, 2005.

[11] K. A. Selvakumaran, S. D. Purohit, and A. Secer, "Majorization for a class of analytic functions defined by $q$-differentiation," Mathematical Problems in Engineering, vol. 2014, Article ID 653917, 5 pages, 2014.

[12] H. M. Srivastava and J. Choi, Zeta and q-Zeta Functions and Associated Series and Integrals, Elsevier Science, New York, NY, USA, 2006.

[13] H. Zhou, K. A. Selvakumaran, K. A. Selvakumaran, S. Sivasubramanian, S. D. Purohit, and H. Tang, "Subordination problems for a new class of Bazilevič functions associated with $k$-symmetric points and fractional $q$-calculus operators," AIMS Mathematics, vol. 6, no. 8, pp. 8642-8653, 2021.

[14] F. H. Jackson, "The basic Gamma-function and the elliptic functions," Proceedings of the Royal Society of London. Series A, Containing Papers of a Mathematical and Physical Character, vol. 76, no. 508, pp. 127-144, 1905.

[15] G. Gasper and M. Rahman, Basic Hypergeometric Series, Encyclopedia of Mathematics and its Applications 35, Cambridge University Press, Cambridge, UK, 1990.

[16] M. Garg and L. Chanchalani, "Kober fractional $q$-derivative operators," Le Matematiche, vol. 16, pp. 13-26, 2011.

[17] Y. Luchko and J. J. Trujillo, "Caputo-type modification of the Erdely-Kober fractional derivative," Fractional Calculus and Applied Analysis, vol. 10, pp. 249-267, 2007. 